\title{
Acute necrotizing encephalopathy with organic psychosis: a pediatric case report
}

\author{
Leman Tekin Orgun $^{1 \oplus}$, Ebru Arhan ${ }^{1 \oplus}$, Kürşad Aydın $^{1 \oplus}$, Yasemin Taş Torun ${ }^{2 \oplus}$, \\ Esra Güney ${ }^{2 \oplus}$, Ayşe Serdaroğlu ${ }^{1 \oplus}$ \\ Departments of ${ }^{1}$ Pediatric Neurology and ${ }^{2}$ Child and Adolescent Psychiatry, Gazi University Faculty of Medicine, Ankara, Turkey.
}

\begin{abstract}
Background. Enteroviruses-associated acute necrotizing encephalopathy (ANE) of childhood is rarely reported in the literature. Clinico-radiological features of ANE are well-recognized and bilateral thalamic nuclei are frequently affected by ANE. Neuropsychiatric symptoms are rarely seen. Thalamic damage can cause psychosis.

Case. Herein, we present a pediatric case of enterovirus-associated ANE presenting with psychosis related to thalamus damage in whom a favorable response to treatment was achieved.

Conclusion. Thalamic damage occurs during the Enteroviruses-associated acute necrotizing encephalopathy and it can be related psychiatric sympthoms. Clinicians should be aware of uncommon presentations of ANE, and patients with thalamic damage should be followed for neuropsychiatric manifestations. Early recognition and appropriate treatment of ANE is important to obtain favorable outcomes.
\end{abstract}

Key words: childhood, encephalopathy, enterovirus, psychosis, thalamus damage.

Acute necrotizing encephalopathy (ANE) of childhood is a discrete form of acute encephalopathy induced by acute febrile diseases with a high morbidity and mortality rate, and predominantly affects infants and young children. ${ }^{1}$ The main presenting symptoms of ANE include fever, vomiting, seizures, acute encephalopathy, and rapid alteration of consciousness after a non-specific viral illness. ${ }^{2}$ Although the etiology and pathogenesis of ANE have not been explained clearly yet, it usually develops secondary to viral infections. ${ }^{3}$ The influenza virus, parainfluenza virus, and human herpesvirus-6 (HHV-6) infections are the most common etiological agents, whereas enteroviruses are rarely reported to be the cause of ANE. ${ }^{1}$

$\bowtie$ Leman Tekin Orgun

lemantekin15@yahoo.com

Received 18th February 2019, revised 6th April 2019, 14th May 2019, accepted 7th July 2019.

This case report was presented as a poster presentation at the 18th Annual Congres on Pediatric Neurology, 20-24 April 2016, Antalya, Turkey.
To date ANE has been recognized primarily as a clinic-radiological disorder the lesions involve multiple brain areas, including the brainstem, periventricular white matter, internal capsule, putamen, cerebellum and bilateral thalamic nuclei, which are known to be the most affected areas in ANE. ${ }^{2-4}$ Thalamic damage may occur during various viral infections, vascular pathologies or tumoral infiltration and can cause perception of pain, movement disorders, insomnia and other sleep disorders, and psychosis. $^{5}$ However, neuropsychiatric symptoms during ANE are reported very rarely ${ }^{6}$

In this report, we described a pediatric case of possible enterovirus-associated ANE presented with psychosis in whom a favorable response to immunotherapy was achieved.

\section{Case Report}

A 17-year-old, previously healthy male patient presented to our emergency department with a sudden-onset weakness of the left hand and 
speech impairment. He was on paracetamol treatment for two days due to fever, rhinorrhea, and headache. His medical history was negative for recent travels and exposure to other drugs, and he was fully immunized. His family history revealed no neurological disorders. Physical examination findings were normal, except for pharyngeal hyperemia and fever. Neurological examination showed dysarthria, decreased muscle strength $(4 / 5)$ on the left upper limb, and a Glasgow Coma Scale score of 13/15. He was hospitalized with the suspicion of encephalitis. He had a rapid deterioration of the muscle strength which decreased to $2 / 5$ on the left upper limb and $4 / 5$ on the right upper limb with a decreased level of consciousness and encephalopathy which developed within a few hours. He was taken to the intensive care unit, and treatment with oseltamivir, acyclovir, and intravenous immunoglobulin (IVIG) was initiated. Laboratory analysis showed normal blood counts, blood chemistry, electrolytes, thyroid hormone, thyroid antibodies, and vitamin $\mathrm{B}_{12}$ levels. Metabolic studies including blood $\mathrm{pH}$, bicarbonate, lactate, ammonia, and tandem metabolic screening were normal. C-reactive protein level (CRP), erythrocyte sedimentation rate (ESR), and immunoglobulin profile, C3 and C4 levels, antinuclear antibody, and anti-DNA antibodies were also normal. Cerebrospinal fluid (CSF) examination showed normal glucose and protein levels without pleocytosis (protein: $30 \mathrm{mg} / \mathrm{dL}$ [reference:15-40 $\mathrm{mg} / \mathrm{dL}$ ], glucose: $60 \mathrm{mg} / \mathrm{dL}$ [reference: 40-70 $\mathrm{mg} / \mathrm{dL}$, and blood glucose level of $82 \mathrm{mg} / \mathrm{dL}$ ). The CSF Gram staining and culture results were negative and the polymerase chain reaction (PCR) test of the CSF for herpes simplex virus-1 and -2 (HSV-1 and HSV-2), EpsteinBarr virus (EBV), cytomegalovirus (CMV), non-polio enteroviruses and mycoplasma was negative. Electroencephalography showed diffuse slowing of $5-6 \mathrm{~Hz}$ theta wave without epileptiform activity. On brain magnetic resonance imaging (MRI), T2weighted imaging (T2WI) revealed symmetric, heterogeneous hyperintense signal changes on the thalamic nuclei, and decreased signal intensities in the center which are surrounded by increased signal intensities on the anterior thalamus (Fig. 1). The susceptibility-weighted imaging (SWI) showed multiple petechial hemorrhagic areas in thalamus. However, MRI angiography and spinal cord MRI were normal. The diagnosis of ANE was established by clinical and radiological features. He was treated by immunotherapy (total $2 \mathrm{~g} / \mathrm{kg}$ IVIG within five days and 1,000 mg/day intravenous [IV] pulse methylprednisolone). He showed a rapid recovery on the fourth day of treatment. His muscle strength became normal in all extremities, except for fine motor skills on his left hand. Throat swap PCR which was obtained on the first day of hospitalization was positive for enterovirus. PCR for influenza virus, parainfluenza virus, and HHV-6 were negative. On the fifth day of hospitalization, the patient developed nervousness, auditory and visual hallucinations, behavioral changes, intensive anxiety and treatment incompatibility. He was, then, evaluated by a child and adolescent psychiatrist and was diagnosed with psychosis (Brief Psychiatric Rating Scale [BPRS] score: 66) due to his overall condition, treatment with haloperidol $2 \mathrm{mg} /$ day was initiated and the dose was titrated, if necessary.

On Day 17, he was discharged without any motor deficit and with mild psychiatric complaints (BPRS score: 12), with haloperidol $2 \mathrm{mg} /$ day treatment. After five days of discharge, he was admitted to the emergency department due to nonsense speech, amnesia, severe agitation, and self-mutilation behaviors. Psychiatric consultation showed increased psychotic symptoms (BPRS score: 68), and dose of haloperidol was increased to $5 \mathrm{mg}$ /day. On Day 22, follow-up MRI showed moderate regression in both thalamic lesions. In addition, psychiatric symptoms improved and dose of haloperidol treatment was reduced to $3 \mathrm{mg} /$ day. On Day 8 of follow-up, he was discharged with significant clinical and psychiatric improvements (BPRS score: 8) and haloperidol $2 \mathrm{mg} /$ day was prescribed. Haloperidol treatment was discontinued one month later. At 


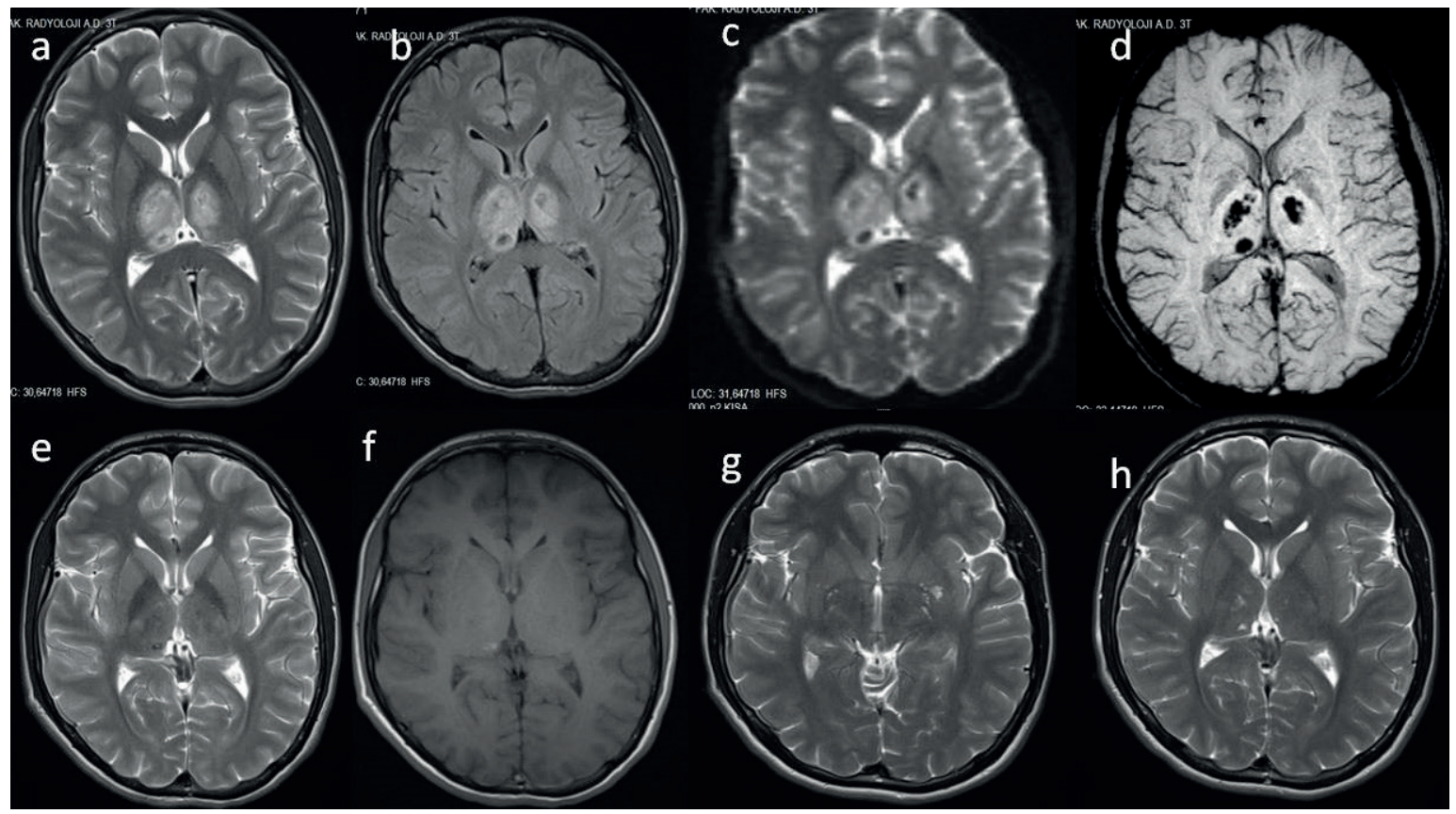

Fig. 1. Dynamic changes of magnetic resonance imaging (MRI) of the case . (a) and, (b) and (c) were, respectively, the T2-weighted image(T2WI), fluid attenuated inversion recovery(FLAIR), and diffusion-weighted imaging(DWI) at onset which revealed symmetric, heterogeneous hyperintense signal changes on the thalamic nuclei, and decreased signal intensities in the center which are surrounded by increased signal intensities on the anterior thalamus, and (d)susceptibility-weighted imaging(SWI) at onset which showed multiple petechial hemorrhagic areas in thalamus. (e), and (f) were, respectively, the T2WI, and T1WI imaging at $22^{\text {th }}$ day, which revealed gradually regression of edema and mass effect in both thalamic lesions. (g) and (h) were T2WI imaging of fallow up at 18 months which showed mild sequelae and necrotic changes on bilateral thalamic nuclei.

two months, repeated MRI showed significant regression in both thalamic lesions with normal neurological and psychiatric examination findings. At 18 months, repeated MRI showed mild sequelae and necrotic changes on bilateral thalamic nuclei. At the final visit, his neurological and psychiatric examination findings were all normal. Informed consent was taken from patient and patient's parents.

\section{Discussion}

In this report, we described a pediatric case of possible enterovirus-associated ANE presenting with psychosis with a favorite response to immunotherapy. ANE frequently develops secondary to viral infections; influenza viruses, parainfluenza viruses, and HHV-6 infections are the most common etiological agents of ANE in children. ${ }^{3}$ However, there are only few patients with ANE associated with enterovirus infection. ${ }^{1}$

Enterovirus is a well-known neurotropic virus which causes aseptic meningitis, encephalitis, brainstem encephalitis, and acute flaccid paralysis. ${ }^{1,7}$ Although enterovirus-associated ANE may present with a wide range of clinical features, psychiatric symptoms have been rarely reported. ${ }^{7}$ The clinical manifestations of encephalitis due to viral etiology are extremely variable and reflect the specific area and degree of brain involvement and inherent pathogenicity of the offending agent. ${ }^{8}$ Enterovirus encephalitis (EVE) predominantly manifests as a generalized syndrome, with nonspecific alterations in consciousness ranging from lethargy and mild disorientation to coma. Clinical features of EVE are more commonly related to brainstem, frontal lobe, temporal lobe, and thalamus manifestations. ${ }^{8}$ Other 
viral agents involving thalamus are EBV, and influenza virus. ${ }^{8}$ Among viral etiologic agents other than enterovirus, herpes simplex virus encephalitis (HSVE) presents itself with atypical manifestations in children. Besides, encephalitis associated with primary varicella Zoster Virus (VZV) infection may develop as a nonspecific syndrome or as acute or postinfectious CNS disease or with isolated symptoms such as acute cerebellar ataxia. In the absence of skin lesions, it is difficult to diagnose VZV encephalitis. In cases due to influenza-associated encephalitis, neurologic signs are observed several days after the onset of upper respiratory tract symptoms. ${ }^{8}$

Neuroradiological features of ANE include multifocal, symmetric brain lesions involving the thalami, brainstem, cerebral white matter, and cerebellum. ${ }^{2}$ Bilateral thalamic nuclei are typically involved in all patients with ANE, which is known as a distinctive feature of ANE. ${ }^{3,9}$ The pathophysiological changes in ANE include edema, petechial hemorrhage, and necrosis. ${ }^{2}$ Generally in ANE, increased signal intensities are detected in the center of the lesions which are surrounded by the decreased signals on T1-weighted imaging (T1WI). T2-weighted imaging (T2WI) may reveal decreased signal intensities that are surrounded by increased or homogeneous increased signal intensities. The classical neuroimaging is "concentric/laminar structure" or "tricolor pattern" or target-like appearance. ${ }^{3}$ In HSVE, neuroimaging studies with MRI reveal asymmetric hyperintense lesions on T2weighted sequences at edematous areas in the mesiotemporal and orbitofrontal lobes and the insular cortex. Among early neuroradiologic menifestations, there is diffusion restriction on diffusion-weighted imaging (DWI) early in the course of HSVE. ${ }^{10}$ It is reported that features of inflammation in EVE can be clearly determined particularly in the anterior horns of spinal cord, the dorsal pons, and the medulla on MRI. ${ }^{11}$

T1-weighted MRI of the present patient showed heterogeneous and decreased signal intensities, while T2WI revealed symmetric, heterogeneous, and hyperintense signal changes on the thalamic nuclei with decreased signal intensities in the center surrounded by increased signal intensities on the anterior thalamus. The SWI also showed multiple petechial hemorrhages, which were more prominent on the right thalamus.

The thalamus is a highly connected subcortical structure which integrates sensory and cortical information. ${ }^{12}$ It is critical for increased awareness and cognition. Thalamic dysfunction may result from several viral infections or vascular pathologies, and can cause perception of pain, movement disorders, sleep disorders, and psychosis. ${ }^{5}$ However, it is still unknown whether these findings present in different psychotic disorders and -regions, and how they relate to structural thalamic alterations. ${ }^{12}$ The thalamocortical circuits are implicated in mood and psychotic disorders. Thalamo-cortical dysrhythmia (TCD) has attracted increasing attention as an underlying mechanism for psychiatric disorders. In TCD, persistent thalamic delta and/or theta range activity serves as the trigger for thalamo-cortical dysfunction. TCD has been proposed to be related to the dysfunction of low-voltage calcium channels. ${ }^{13,14}$ Our case had psychotic symptoms including irritability, auditory and visual hallucinations, and intense agitation which might be compatible with the MRI findings with edema and multiple hemorrhagic areas in bilateral thalamic nuclei. We hypothesized that thalamic damage might lead to TCD and TCD possibly underpinned our patient's behavioral disturbances and personality changes.

The critical care follow-up and treatment are essential tools for management of all ANE patients as in other acute encephalopathies. ${ }^{1}$ IVIG was recommended for the treatment of patients with encephalitis by the World Health Organization guide about hand-footmouth disease..$^{15}$ According to this guide, IVIG was recommended in treatment of patients with encephalitis. In the literature, treatment with antiviral drugs, methylprednisolone, dexamethasone, plasmapheresis, antithrombin III and therapeutic hypothermia were also tried 
on ANE patients. ${ }^{3,16}$ There is still a debate about the use of IV steroids in ANE. It is indicated in the literature that both steroid and IVIG may be given in ANE. ${ }^{3,15,16}$ Most recent studies have suggested IV steroids for ANE.1,17 The role of steroid therapy in ANE is controversial. Our patient was treated with supportive measures and IVIG in the acute phase. Steroid was also added after the exclusion of active viral infection with laboratory testing. Therefore, IVIG and pulse methyl prednisolone were administered simultaneously in our case. Although steroids may cause psychosis, psychiatric side effects with corticosteroids appear to be dose-dependent. ${ }^{18}$ In the literature, it was reported that steroid-induced psychosis was more frequent in patients whom $40 \mathrm{mg} /$ day prednisolone was administered, among women, and during the first two weeks of steroid treatment. ${ }^{18}$

Specific diagnosis of EV meningitis depends on EV isolation from CSF. The specificity of the method is only $65-75 \%$ due to low titers of EV serotypes in CSF. ${ }^{19} \mathrm{EV}$ isolation from oropharynx or feces is also valuable for diagnosis in patients with aseptic meningitis. After infection, EV may be present in oropharynx for one week, and in feces for weeks. ${ }^{20}$ It is difficult to diagnose EV infection by using the immunoassay method, because there is no common antigen between different serotypes, and the virüs has low concentration in body fluids. ${ }^{20}$ It is difficult to grow EV serotypes in cell culture. However, EV "reverse transcription PCR" is reported as a faster and sensitive diagnostic method in clinically critical time-frame. Therefore, PCR, with $94-100 \%$ diagnostic specificity, is the new gold standard for EV detection. ${ }^{21}$ Moreover, presence of $\mathrm{EV}$ has been shown in healthy individuals during epidemics. ${ }^{22} \mathrm{EV}$ isolation from a source other than CSF or blood may indicate a previous infection. In the present case, it was concluded that ANE was due to $\mathrm{EV}$ because of the isolation of EV only from oropharyngeal swab.
The prognosis of ANE varies from complete recovery to death. ${ }^{3}$ The mortality rate is about $30 \%$ and less than $10 \%$ of patients recover completely, although significant neurological problems are frequent in survivors. ${ }^{2,23}$ In a large cohort, it was reported that prognosis of encephalitis in pediatric population was not promising; only one third of cases had incomplete recovery whereas $6.8 \%$ of patients died. ${ }^{8}$ Prognosis depends mainly on the infectious agents. According to the literature, more than $35 \%$ of patients with HSVE recover with severe sequela or die. Early and late relapses were reported up to $26 \%$ of children who had HSV-1 encephalitis. In EBV encephalitis, persistent neurological deficits were observed less. In Japan and Taiwan, high mortality rates were reported in ANE associated with influenza infection. ${ }^{8}$ Concrete data about the remaining viral etiologic agents is limited, because they were reported as case reports and small series. ${ }^{8}$ In a large multicenter study conducted on Chinese children with viral encephalitis and meningitis, EVE is reported as the most common etiologic agent both in acute encephalitis and meningitis with the incidence of sequeala and fatality rate of $7.5 \%$ and $0.8 \%$, respectively. ${ }^{24}$ Our case recovered without any neurological sequelae. We, therefore, consider that early diagnosis and early steroid treatment played a critical role in our case in whom a favorable response was achieved.

In conclusion, early recognition and appropriate treatment after early diagnosis of ANE is of utmost important to obtain favorable outcomes. Although enterovirus is known as a rare pathogen for ANE, the laboratories should have tests for it in suspected cases. In addition, clinicians should be aware of this uncommon presentation of ANE, and patients with thalamic damage should be followed for neuropsychiatric manifestations. 


\section{REFERENCES}

1. Tabarki B, Thabet F, Al Shafi S, Al Adwani N, Chehab $\mathrm{M}, \mathrm{Al}$ Shahwan S. Acute necrotizing encephalopathy associated with enterovirus infection. Brain Dev 2013; 35: 454-457.

2. Mizuguchi M. Acute necrotizing encephalopathy of childhood: a novel form of acute encephalopathy prevalent in Japan and Taiwan. Brain Dev 1997; 19: 81-92.

3. Wu X, Wu W, Pan W, Wu L, Liu K, Zhang HL. Acute necrotizing encephalopathy: an underrecognized clinicoradiologic disorder. Mediators Inflamm 2015; 2015: 792578.

4. Voudris KA, Skaardoutsou A, Haronitis I, Vagiakou EA, Zeis PM. Brain MRI findings in influenza A-associated acute necrotizing encephalopathy of childhood. Eur J Paediatr Neurol 2001; 5: 199-202.

5. De Witte L, Brouns R, Kavadias D, Engelborghs S, De Deyn PP, Marien P. Cognitive, affective and behavioural disturbances following vascular thalamic lesions: a review. Cortex 2011; 47: 273-319.

6. Partlow GD, del Carpio-O'Donovan R, Melanson D, Peters TM. Bilateral thalamic glioma: review of eight cases with personality change and mental deterioration. AJNR Am J Neuroradiol 1992; 13: 1225-1230.

7. Okumura A, Mizuguchi M, Aiba H, Tanabe T, Tsuji $\mathrm{T}$, Ohno A. Delirious behavior in children with acute necrotizing encephalopathy. Brain Dev 2009; 31: 594-599.

8. Glaser CA, Bloch KC. Encephalitis. In: Long SS, Prober CG, Fischer M (eds). Principles and Practice of Pediatric Infectious Diseases (5th ed). Philadelphia: Elsevier, 2018: 305-322.

9. Ravid S, Topper L, Eviatar L. Acute necrotizing encephalopathy presenting as a basal ganglia syndrome. J Child Neurol 2001; 16: 461-462.

10. Bradshaw MJ, Venkatesan A. Herpes simplex virus-1 encephalitis in adults: pathophysiology, diagnosis, and management. Neurotherapeutics 2016; 13: 493508.

11. Ooi MH, Wong SC, Lewthwaite P, Cardosa MJ, Solomon T. Clinical features, diagnosis, and management of enterovirus 71. Lancet Neurol 2010; 9: 1097-1105.

12. Fowlkes AL, Honarmand S, Glaser C, et al. Enterovirus-associated encephalitis in the California encephalitis project, 1998-2005. J Infect Dis 2008; 198: 1685-1691.
13. McGilchrist I, Goldstein LH, Jadresic D, Fenwick P. Thalamo-frontal psychosis. Br J Psychiatry 1993; 163: 113-115.

14. Schulman JJ, Cancro R, Lowe S, Lu F, Walton KD, Llinás RR. Imaging of thalamocortical dysrhythmia in neuropsychiatry. Front Hum Neurosci 2011; 5: 69.

15. Cardosa J, Farrar J, Zijian F, et al. A Guide to Clinical Management and Public Health Response for Hand, Foot and Mouth Disease (HFMD). Geneva, Switzerland: World Health Organization Western Pacific Region, 2011.

16. Yamamoto H, Okumura A, Natsume J, Kojima S, Mizuguchi M. A severity score for acute necrotizing encephalopathy. Brain Dev 2015; 37: 322-327.

17. Kim JH, Kim IO, Lim MK, et al. Acute necrotizing encephalopathy in Korean infants and children: imaging findings and diverse clinical outcome. Korean J Radiol 2004; 5: 171-177.

18. Skatun KC, Kaufmann T, Brandt CL, et al. Thalamocortical functional connectivity in schizophrenia and bipolar disorder. Brain Imaging Behav 2018; 12: 640652.

19. Romero JR.Viral meningitis and the aseptic meningitis syndrome. In: Scheld WM, Whitley RJ, Marra CM, (eds). Infections of the Central Nervous System (4th ed). Philadelphia: Wolters Kluwer Health, 2014: 65-83.

20. Tunkel AR, Beek DV, Scheld WM. Acute meningitis. In: Bennett JE, Dolin R, Blaser MJ (eds). Mandell, Douglas, and Bennett's Principles and Practice of Infectious Diseases (8th ed). Vol.1. Philadelphia: Churchill Livingstone Elsevier, 2015: 1097-1137.

21. Robinson CC, Willis M, Meagher A, Gieseker KE, Rotbart H, Glodé MP. Impact of rapid polymerase chain reaction results on management of pediatric patients with enteroviral meningitis. Pediatr Infect Dis J 2002; 21: 283-286.

22. Connoly KJ, Hammer SM. The acute aseptic meningitis syndrome. Infect Dis Clin North Am 1990; 4: 599-622.

23. Okumura A, Mizuguchi M, Aiba H, Tanabe T, Tsuji T, Ohno A. Delirious behavior in children with acute necrotizing encephalopathy. Brain Dev 2009; 31: 594-599.

24. Ai J, Xie Z, Liu G, et al. Etiology and prognosis of acute viral encephalitis and meningitis in Chinese children: a multicentre prospective study. BMC Infect Dis 2017; 17: 494. 